\title{
Análisis de neuroeconomía como nuevo paradigma en la ciencia económica
}

Analysis of neuroeconomics as a new paradigm

in economic science

Denise Elisabet Arias

Facultad de Ciencias Económicas,

Universidad Nacional del Litoral,

Argentina.

E-mail:deniseelisabetarias@gmail.com

Fecha de recepción: 01/07/2016 Fecha de aprobación: 21/11/2016

\section{Resumen}

La neuroeconomía es una reciente disciplina que estudia lo que sucede en el cerebro humano durante la toma de decisiones, y la relación entre las emociones y la conducta de los agentes económicos. Como su nombre lo indica, surge de la conjunción entre neurociencias y economía. En los últimos años el avance vertiginoso de las neurociencias permitió la realización de numerosas investigaciones en esta área, haciendo posibles nuevos enfoques en los análisis económicos.

En el año 2002 se otorgó el Premio Nobel de Economía al psicólogo israelí Daniel Kahneman, quien en su estudio reveló que se cometen errores a la hora de tomar decisiones económicas. Fue pionero en integrar descubrimientos de psicología en las ciencias económicas y demostró que el supuesto clásico de la decisión racional del consumidor es afectado por importantes sesgos cognitivos.

Se cuestiona la teoría económica clásica desde Adam Smith que se basa en que los miembros de la sociedad son siempre racionales, definidos como «homo economicus» que buscan maximizar las utilidades, analizando objetivamente los costos y beneficios.

Palabras clave

- neuroeconomía

- economía experimental

- economía conductual

- racionalidad limitada

- homo economicus
En el presente trabajo se hace una revisión de los aportes clásicos de la economía y de cómo el conocimiento detallado del cerebro humano y sus funciones logró que las neurociencias puedan aplicar esos nuevos saberes en su campo, teniendo en cuenta que los aspectos emocionales y racionales del comportamiento son derivados del mismo cerebro. 
Keywords

- neuroeconomics

- experimental economics

- behavioral economics

- limited rationality

- homo economicus

\section{Abstract}

Neuroeconomics is a recent discipline which studies what happens in the human brain during decision making, and the relationship between emotions and the behavior of economic agents.

As its name indicates it arises from the conjunction between neuroscience and economics. During the last years, the rapid advance of the neurosciences allowed the realization of numerous investigations in this area, making possible new approaches in the economic analysis.

In 2002 the Nobel Prize in Economics was given to Israeli psychologist Daniel Kahneman, who in his study revealed that mistakes are made in making economical decisions. He was a pioneer in integrating discoveries of psychology in the economic sciences, and showed that the classical assumption of rational consumer decision is affected by important cognitive biases.

Adam Smith's classical economic theory is questioned, which is based on the fact that the members of society are always rational, defining them as "homo economicus" who seek to maximize profits by objectively analyzing costs and benefits.

In the present work a review of the classic contributions of the economy is made, and how the detailed knowledge of the human brain and its functions managed that the neurosciences can apply this new knowledge in its field, taking into account that the emotional and rational aspects of the behavior are derived from the same brain.

\section{Primeros interrogantes en los clásicos}

El modelo económico neoclásico tiene como fundamento que los agentes económicos actúan racionalmente, y por lo tanto optimizan su utilidad de manera previsible cuando consumen y producen eficientemente al combinar de la mejor manera posible los factores de producción. No se autoengañan y pasan a ser virtuosos de las estadísticas actuando en pos de objetivos claros y conscientes.

A pesar de ello se han realizado experimentos que reúnen evidencia de cómo las personas se apartan de la razón pura en sus decisiones. Se observaron comportamientos de la conducta cotidiana; por ejemplo, si una persona quiere comprar manzanas y en la misma cuadra de su casa venden a $\$ 60$ el kilo, pero a 5 cuadras lo venden a $\$ 30$, iiría caminando esas 5 cuadras para ahorrar $\$ 30$ ? Muy probablemente, sí. Ahora esa misma persona se encuentra en un local para comprar una remera, una cuesta $\$ 310$ y otra $\$ 340$, iserán esos $\$ 30$ cruciales para la decisión de la compra? Probablemente, no. Aunque siempre se trate de $\$ 30$, en el primer caso estará dispuesta a hacer el esfuerzo para ahorrarlos y en el segundo caso no le da importancia. De la misma forma se observó cómo funciona el mercado en los diferentes días de la semana 0 en los distintos meses del año, encontrándose el «efecto viernes» 0 el «efecto enero», que son factores que se dificultan explicar desde el «homo economicus» (Campanario, 2005).

Día a día tomamos decisiones, como comprar una entrada al cine 0 al teatro, y otras que podrían marcar el rumbo de un negocio. La pregunta es qué nos lleva a tomar cada una de ellas. Ya sean 
pequeñas 0 grandes decisiones, se observa que en realidad la mayoría de las veces somos menos racionales de lo que creemos y que las emociones siempre están presentes, consciente 0 inconscientemente, jugando un rol muy importante en el comportamiento social.

No hay que perder de vista que la economía es una ciencia social que depende del comportamiento del hombre y por eso muchas veces es impredecible. Aunque algunos economistas digan que es la más exacta dentro de las ciencias sociales, no deja de ser el hombre quien debe estudiarse a sí mismo.

Mochón y Becker al definir la economía nos dicen que «es la ciencia que estudia la asignación más conveniente de los recursos escasos de una sociedad para la obtención de un conjunto ordenado de objetivos» (Mochón y Becker, 1997 en Perticarari y Hauque, 2013:86). Podemos afirmar que se ocupa de cuestiones que surgen en relación con la satisfacción de necesidades, interesándose en la forma en que se administran los recursos escasos, la producción de bienes y la distribución a todos los miembros de la sociedad. Ofrece un método que permite ordenar y establecer prioridades al momento de tomar decisiones sobre las necesidades individuales y colectivas. Es por ello que algunos autores la denominan la «ciencia de la elección» (Mochón y Becker, 1997).

Adam Smith en su escrito La Riqueza de las Naciones dice: «No esperamos nuestra cena de benevolencia del carnicero, el cervecero o el panadero, sino del respeto que sienten por sus propios intereses» (Perticarari y Hauque, 2013:49). Es así que supuestamente los seres humanos actuamos en interés propio y de manera racional, aunque él mismo deja abierta una inconsistencia en esta cuestión.

Los modelos económicos tienen presente el «homo economicus», pero ya Smtih aclaraba en sus obras que en la mente se descubren las reglas generales y valores propios según los cuales normamos nuestros actos, y es el lugar donde depositamos ideas que nos llevan a modelar nuestro comportamiento. También expone que es absurdo e inteligible suponer que las conductas humanas provienen exclusivamente de la razón, siendo que se involucran en ellas sentimientos y emociones. En su obra la Teoría de los Sentimientos Morales explica lo siguiente: «La razón no puede hacer que un objeto resulte por sí mismo agradable o desagradable; la razón sólo puede revelar que tal objeto es medio para obtener algo que sea placentero o no, y de este modo puede hacer que el objeto, por consideración a esa otra cosa, nos resulte agradable o desagradable. Más nada puede ser agradable 0 desagradable por sí mismo, que no sea porque así nos lo presenta un inmediato sentido y sensación» (Smith, 1941:148).

El padre de la economía ya le estaba dando importancia a los procesos mentales para la toma de decisiones, para guiar nuestras preferencias, nuestras elecciones a determinados bienes, y para condicionar nuestro actuar. Por mucho tiempo en la economía se dejó esta cuestión de lado, pero hoy, a través de las neurociencias, podemos encontrar qué hay detrás de nuestros comportamientos, qué sucede en nuestro cerebro antes de tomar una decisión, cuáles son los factores de placer y cómo se desencadenan.

J. M. Keynes cuestionaba cómo podía ser que aun cuando el análisis lógico de los proyectos de inversión mostraban su inconveniencia, los agentes económicos decidían invertir a pesar de ser alta la probabilidad de que el proyecto no resulte rentable. En su libro Teoría general de la ocupación, el interés y el dinero utiliza el concepto denominado «animal spirit» dejando de lado la pura racionalidad, ya que la conducta humana demuestra un deseo de hacer cosas y emprender tareas, pero que muchas veces si se piensan sólo desde lo racional no se llevarían a cabo. Esos deseos de actuar no son constantes, sino que en algunos periodos tienen más fuerza que en otros. Los «animal spirits» se aproximan a ondas de optimismo y pesimismo que dan vueltas en la sociedad y nos mueven a la acción simplemente por el placer que produce la acción. Tiene en cuenta que las personas podemos ser emocionales, olvidadizas e impulsivas (Navarro, 2007). 
Keynes también expone la idea de que si en una empresa el optimismo y la fogosidad se enfrían y se deja como única base de sustentación la previsión matemática, la empresa se marchitará y morirá.

\section{Emociones y neurociencias}

La emoción es un concepto que en economía muchas veces no se tomó en cuenta porque da la sensación de que la emoción desaparece frente al pensamiento lógico, siendo que en realidad las decisiones que tomamos pensando que son racionales están llenas de emociones. Lo racional no sustituye a lo emocional, sino que lo complementa e incide en su desarrollo y en cada decisión final que tomamos.

Por ejemplo, al analizar la música, ya que genera emociones en cualquier persona, se observa que primero sus elementos son racionalizados y se estudian las combinaciones y los efectos que generan. Se procede a escribirla para que al ser ejecutada se produzca una emoción y se obtenga la conducta deseada. De esta forma, si se quiere una conducta pasiva y de tranquilidad en el oyente basta con hacer acordes menores que transmitan tristeza 0 melancolía, demostrando que la conducta aparece desde la emoción y que se pueden racionalizar los pasos para llegar a ella.

Al utilizar los avances de las neurociencias sobre las emociones es posible determinar mecanismos que subyacen a la conducta, y que impulsen a actuar de determinada manera en algunas circunstancias y a no hacerlo en otras.

En las neurociencias existe un campo interdisciplinario que se dedica al estudio del sistema nervioso. Se aúnan esfuerzos para conseguir el objetivo en común que consiste en entender mejor cómo percibimos, aprendemos, recordamos, pensamos y sentimos.

Su desarrollo fue posible a partir de la década de 1950 cuando disciplinas hasta entonces independientes, como la neuroanatomía, neuroquímica y el estudio de la conducta, fueron gradualmente fusio- nándose. En la década del 80 al estudio interdisciplinario del sistema nervioso se le suman otras áreas como la biología molecular, la genética molecular y luego la neurobiología de sistemas y la psicología cognitiva (Álvarez Gonzalez y Trágapa Ortega, 2005).

Para estudiar algunas cargas genéticas que traemos y que condicionan nuestra conducta se realizan investigaciones con animales; para eso el abordaje combinado de monos y humanos es un recurso muy utilizado. En la Universidad de Emory, Estados Unidos, se realizó un experimento con monos capuchinos marrones que son considerados entre los animales más inteligentes. Se les daba para comer cocos, luego de un tiempo se dividió al grupo en dos, y a una parte se le comenzó a dar uvas, mientras que a la otra se le siguió dando cocos. Las uvas para ellos son consideradas bienes suntuarios y mucho más preferibles que los cocos. El resultado fue que los que seguían recibiendo cocos, al ver que los demás recibían uvas, tiraban furiosamente sus cocos reaccionando ante lo que ellos consideraban injusto.

El sentimiento de justicia está presente en los humanos también, y se lo demostró con un experimento llamado el juego del ultimátum. En este juego participan dos personas, se le da dinero a un jugador (A) y al otro jugador (B) no se le entrega nada. Se le dice al primer jugador que debe darle una parte al jugador B que no recibió nada, y este último tendrá las opciones de aceptar o rechazar lo que se le ofrece. Si lo rechaza ninguno de los dos obtendrá algo. Lógicamente, según la teoría económica convencional que supone que las personas son maximizadoras racionales de la riqueza, podemos decir que el segundo jugador aceptará cualquier cantidad de dinero que A quiera darle, ya que un peso es mejor que nada. Pero los resultados difieren de esta predicción. Ocurría que si A le ofrecía al jugador B menos del $20 \%$, optaba por rechazarlo y ninguno recibía nada, prefiriendo penalizarlo por considerarlo injusto. También se observó que lo que el primer jugador ofrecía era en general al rededor del $40 \%$. Estas posturas demostraron el 
sentimiento de justicia que prevalece en la toma de decisiones (Campanario, 2005).

Hoy se cree que la sensibilidad por la justicia es una mezcla de factores culturales y genéticos, y se observa en la economía de la vida cotidiana, en el hogar y en las empresas. En un análisis de la posmodernidad algunos economistas han sugerido que la justicia percibida de lo que la empresa le paga a sus trabajadores debe entrar en el panorama de análisis económico de la misma, ya que en un año excepcionalmente rentable, los trabajadores esperarán que se les pague una justa proporción, de otro modo castigarán a la empresa ya sea reduciendo el esfuerzo, con huelgas e incluso con actos de vandalismo.

No sólo en la sensibilidad por la justicia, sino también en el conjunto de emociones, algunos científicos opinan que son heredadas y comunes a la especie. Ya Darwin en 1872 planteó la similitud de un conjunto de emociones en todas las civilizaciones. El científico Paul Ekman de Estados Unidos, uno de los psicólogos más destacados del siglo XX, estudió en distintas culturas la interpretación de seis emociones diferentes (alegría, enojo, tristeza, miedo, aversión y sorpresa), y confirmó que cada una de ellas asume un mismo significado, pudiendo decir que éstas son universales.

Sobre las decisiones del mercado laboral un estudio en relación con las preferencias de los trabajadores demuestra que las personas están más alegres con una empresa que otorga un aumento salarial del $10 \%$ en un contexto de inflación del $20 \%$ anual que con otra que reduce los salarios un $5 \%$ en un contexto de estabilidad de precios. Aunque en el primer caso es mayor la pérdida adquisitiva, los trabajadores muestran mayor satisfacción. Esto ocurre porque los seres humanos sufrimos ilusión monetaria y preferimos ganar más, aun cuando la capacidad adquisitiva de ese nuevo salario sea más baja. También tiene que ver con la aversión a las injusticias, porque rechazamos cualquier recorte salarial cuando éste es el resultado deliberado de la acción de una persona que se puede identificar, en este caso el empresario (Tetaz, 2014).

Cuando hay alguien a quien culpar por la pérdida de capacidad adquisitiva el grado de insatisfacción es muy elevado. En cambio cuando nos cuesta identificar al culpable la situación es aceptada con mayor tolerancia. Fastidia más al trabajador la pérdida en su capacidad adquisitiva por la baja de su salario que por el aumento general de precios. Aun cuando la baja de su salario sea del 3 \% y la inflación del 20 \% se va a sentir más molesto por la baja del salario, porque la inflación no es un impuesto legislado y el trabajador no puede determinar con claridad quién lo paga y quién lo cobra.

Otra anomalía a considerar es la aversión a las pérdidas, en donde las personas temen los efectos de las posibles pérdidas más que las posibles ganancias. Por ejemplo si en el trabajo hay noticias acerca de un posible bono extraordinario por un valor de $\$ 1000$ se generarán expectativas y se situará ese valor como punto de referencia. Si luego el bono es de un valor mayor será percibido como ganancia y cualquier valor por debajo será percibido como una pérdida.

\section{Un poco de psicología}

Los comportamientos detallados en los párrafos anteriores nos alejan de lo que haríamos según el «homo economicus», siendo en realidad consecuencias del normal funcionamiento de la mente, y no anomalías o fallas mentales. Por eso, resulta valioso indagar con mayor profundidad la psicología cognitiva. Uno de los primeros científicos sociales en trabajar las fronteras de la economía y de la psicología, el estadounidense Herbert Simon, sugiere que debería considerarse a los humanos no como maximizadores racionales, sino como satisfactores, ya que no siempre eligen el mejor curso de acción posible. Otros economistas de manera similar han sugerido que somos casi racionales 0 que mostramos una racionalidad limitada. 
En su libro expresa que «no debe interpretarse la concentración sobre los aspectos racionales del comportamiento humano como una afirmación de que los seres humanos son siempre, o generalmente, racionales. Este falso concepto, que impregnó la teoría política utilitaria y una gran parte de la teoría económica clásica, ha sido refutado de una manera decisiva por los desarrollos modernos de la psicología y de la sociología» (Simon, 1972:59).

Son útiles los aportes que pueda hacer la psicología, sobre todo desde el punto cognitivo, ya que orienta la atención de los analistas hacia las operaciones mentales y el procesamiento de la información. Años atrás era impensada esta vinculación, ya que la psicología y la economía neoclásica siempre tuvieron visiones distintas del proceso de decisión. Mientras que la psicología se enfoca en entender la naturaleza del proceso y cómo sus elementos son establecidos y modificados por la experiencia de las personas, los modelos económicos estándares asumen que los consumidores e inversores son racionales, actúan con preferencias inmutables y su proceso cognitivo se reduce a maximizar las utilidades. La atención en estos últimos está puesta en las elecciones que hacen las personas y no tanto en cómo llegaron a ellas; en otras palabras, el proceso de decisión para los economistas neoclásicos es una «caja negra». En cambio, la piscología cognitiva permite vincular conductas y emociones, y le da importancia al procesamiento interno de las personas, tiene en cuenta que los humanos somos entidades conscientes de sí mismas, capaces de experimentar distintos tipos de estados psicológicos y de producir comportamientos inteligentes.

H. Simon afirma que en los últimos años se comenzó a lograr una buena comprensión científica de los procesos de información que utilizan los seres humanos en la solución de problemas y en la toma de decisiones. Y que no es de extrañar que se utilicen términos como intuición, perspicacia y criterio, y que estos se envuelvan en el misterio de todo proceso.
Él explica un experimento que se hizo para lograr comprender los procesos de toma de decisiones: se le da a un sujeto un problema, por ejemplo un teorema de geometría euclidiana, y se le pide que lo piense en v0z alta mientras lo resuelve, de esta manera todos sus procesos mentales se llevarán al nivel de la conciencia o se verbalizarán, y de esta manera se espera obtener indicios del cursos que tomarán sus pensamientos. También se graba lo que dice mientras resuelve el problema para luego poder analizarlo en detalle (Simon, 1982).

Al estudiar la grabación se observa que el sujeto compara el problema que le fue dado con otros que conoce y busca similitudes y diferencias, de esta manera surgen subproblemas cuyas soluciones pueden contribuir a la solución del problema principal. Los subproblemas a su vez pueden engendrar nuevos subproblemas, hasta llegar a un problema que pueda resolverse directamente. Luego asciende hasta el nivel superior de problemas y gradualmente comienza a ensamblar resultados que parecerían contribuir a la solución integral del caso.

Aunque no parece complicado, persiste la sensación de que sólo percibimos las partes superficiales del proceso, que existe un gran témpano oculto a nuestra vista y a la conciencia del sujeto.

La neuroeconomía se interesa en esa caja negra y en qué ocurre en el proceso de toma de decisiones para poder analizar efectos económicos. También procura entender qué reacciones físicas y químicas se producen en el cerebro que condicionan las conductas.

\section{Explorando la caja negra}

Gracias al avance tecnológico es posible estudiar la actividad cerebral mientras se realizan tareas cognitivas. Para ello se utiliza la tomografía por emisión de positrones (PET) y la resonancia magnética nuclear funcional (FMRI). Mediante estas técnicas se puede analizar lo que sucede en el interior del cerebro y conocer su actividad en momentos de 
reflexión, de toma de decisiones, cuando memorizamos, mientras dormimos, etc. Son procedimientos incruentos e inofensivos por los cuales se pueden obtener imágenes y determinar cuáles son las áreas que se activan en cada circunstancia debido a la mayor afluencia de sangre. (Navarro, 2007).

Con el desarrollo de estas técnicas y paralelamente al desarrollo de la informática y la utilización de digitalizadores, se lograron avances impensables hace un cuarto de siglo que permitieron un profundo conocimiento del funcionamiento del cerebro humano. La década final del siglo XX se denominó «década del cerebro» y muchos consideraban que el siglo XXI debería llamarse «siglo del cerebro».

El cerebro se adapta continuamente, también se actualiza y se modifica a sí mismo, dependiendo de la función cerebral de que se trate y de la herencia genética, la edad, el género y las experiencias personales. Las neurociencias explican cómo funcionan estos circuitos, cómo se modifican y se relacionan con procesos psíquicos complejos.

Existen análisis de los distintos aspectos que inciden a la hora de tomar decisiones. Por ejemplo, a medida que vamos envejeciendo, algunos neurotransmisores como la dopamina y la serotonina son producidos en menor cantidad, y esto tiene efectos sobre la apreciación de las recompensas y sobre los riesgos que se asumen, siendo los adultos menos propensos a aceptar riesgos a la hora de tomar decisiones. Mediante el uso de FMRI se observa que ciertas áreas del cerebro, como el estrato ventral y partes de la corteza prefrontal, se activan de forma distinta entre los jóvenes y los adultos (Navarro, s.f.).

Hoy sabemos que el cerebro está formado por tres subsistemas: el reptiliano, el límbico y la neocorteza, los cuales interaccionan permanentemente. El cerebro reptiliano es el que controla la conducta automática, el sistema límbico es el responsable de controlar la vida emotiva, y la neocorteza que es la estructura más reciente, está constituida por dos hemisferios cerebrales que son los encargados de los procesos intelectuales superiores. Dado que estas tres partes del cerebro funcionan al mismo tiempo, es lógico considerar lo emocional y lo racional en el mismo nivel a la hora de actuar.

Un trabajo de la Universidad de Yale descubrió que el estrés perjudica la capacidad de planeamiento, juicio y memoria, a través de la generación de una enzima (PKC) que altera la región del cerebro que regula las conductas y las emociones. En Argentina hay estadísticas que muestran que durante la crisis de 2001 y 2002 los hospitales reportaron una multiplicación de la demanda de atención por dolencias que en realidad eran derivadas del estrés. En el Instituto Fleni hay un Servicio de Demanda Espontánea donde los pacientes pueden ser atendidos directamente por un neurólogo, y en ese periodo se quintuplicaron las consultas, mientras que en la Fundación Favaloro aumentaron en $40 \%$ los pacientes con síndrome coronario agudo y se triplicaron las consultas psicoanalíticas (Campanario, 2005).

El neurociéntifico Facundo Manes explica en su charla en TEDx Buenos Aires que en los temas sociales siempre están implicadas las emociones. Señala que la diferencia que tenemos con otras especies es el lóbulo frontal. Esta parte del cerebro tiene neuronas distintas de otras zonas, y es importante en funciones como la planificación, en aspectos racionales, emocionales y de interacción social.

Su función comenzó a estudiarse con el famoso caso de Phineas Gage (1848), quien sufrió un accidente laboral cuando una varilla de acero salió despedida por una explosión, atravesando la zona frontal de su cerebro y saliendo despedida por la parte superior de su cabeza. El operario cayó ensangrentado pero permaneció consciente. Esto último llamó la atención y surgieron los cuestionamientos sobre para qué sirve esa parte del cerebro. Luego de su recuperación se observaron cambios en su personalidad, entre ellos: comenzó a tomar decisiones financieras en contra de sí mismo, decisiones laborales y familiares que no eran las 
mejores, y se observaba en él una gran incapacidad para pensar a largo plazo. No tenía secuelas motoras, y el lenguaje, la memoria y la atención no fueron alterados a pesar del daño. Su capacidad para procesar información tampoco había sido afectada, pero sí su proceso de elección en la toma de decisiones, las cuales estaban basadas en placeres inmediatos, sin lograr medir lo que ocurriría en el futuro. Por esta injuria anatómica en su lóbulo frontal se diagnosticó en P. Gage un déficit emocional (Manes, 2012).

Hoy podemos saber que esta parte del cerebro cumple la importante función de regular las emociones y la razón, y tenemos la certeza de que la toma de decisiones no es un proceso puramente lógico, sino que está ligado por las emociones. El Dr. Manes afirma que se producen emociones y son esas emociones las que guían las conductas (Manes, 2015).

Vivimos tomando decisiones y el cerebro no puede permanentemente analizar los pro y las contras de cada una, por eso evolutivamente realiza un mecanismo automático. Actualmente existe una tendencia a comparar el cerebro con una computadora. Para ello se tiene en cuenta que ambos sistemas tienen las funciones de adquisición, análisis, almacenamiento y generación de la información.

Se presenta la idea de que los seres humanos venimos al mundo equipados con un conjunto de programas innatos que han sido adquiridos por nuestra especie y que a lo largo de los años le han ido brindando ventajas de subsistencia. En términos computacionales suponemos que nuestro hardware es el cerebro y que tenemos el programa preinstalado. Este software es una red neuronal estructurada para manipular símbolos, entonces ante un problema determinado, input, se generan representaciones mentales, a partir de cuyo procesamiento se obtienen soluciones, outputs. En cada persona estas representaciones mentales son clave a la hora de evaluar distintos cursos de acciones posibles.

\section{Otras variables para considerar}

A. Smith también suponía que los seres humanos actuamos movidos por el egoísmo y por la simpatía, y si bien podemos encontrar en el comportamiento egoísta una buena simplificación de los mercados, vemos que él considera el comportamiento altruista. Sabemos que algunas personas pueden ser predominantemente egoístas, pero otras tienen mayor propensión a actuar desinteresadamente, siendo que las relaciones sociales y la organización de la sociedad están ligadas al concepto de altruismo.

La conducta humana es compleja y difícil para encuadrar en fórmulas. El Dr. Alfredo Martín Navarro, miembro de la Academia Nacional de Ciencias Económicas, propone la siguiente función de utilidad a maximizar:

$$
U i=X i+\Sigma j V j(K j i) X j
$$

En donde $\boldsymbol{U}$ es la utilidad del individuo $\boldsymbol{i}, \mathrm{X}$ la utilidad egoísta de individuo $i, V$ es la utilidad que significa para el individuo $i$, la utilidad de los otros $j$ individuos que componen el grupo social, y $K$ el grado de simpatía que el individuo i tiene hacia el individuo $j$, lo que puede tener signo positivo 0 negativo (Navarro, s.f.).

Dado que la solidaridad es un elemento importante en la convivencia social, el estudio de sus fundamentos básicos es de importancia para las ciencias sociales. Se encontraron paralelismos entre centros cerebrales que se activan en respuesta al egoísmo con los que producen el bienestar de las personas.

En los modelos económicos hay otras cuestiones que no son tenidas en cuenta, como el nivel de satisfacción, la memoria y la falta de información. Se tiende a sobreestimar la satisfacción que causan los eventos importantes. Hace unos años en Argentina al cierre del canje, con un proceso de desendeudamiento que marcaría a la economía, se preveía que el día que el país regularizara su deuda externa habría un gran optimismo generalizado. Pero no fue así por mucho tiempo, ya que la preocupación 
por la inflación en 2005 arrasó con cualquier aire de optimismo. Un hallazgo descubierto por la psicología es el «efecto habituación», por el cual para una persona que gana millones en el Quini 6 ese año es el más feliz de su vida, pero por desgracia esa felicidad dura poco y al tiempo vuelve a reportar el mismo nivel de felicidad que tenía antes del acontecimiento. Este fenómeno ocurre tanto en las cosas buenas como las malas. El acostumbramiento a las nuevas condiciones es una característica fundamental que hizo posible la supervivencia del hombre a lo largo de los años (Campanario, 2005).

En cuanto a nuestro sistema de memoria está comprobado que tenemos distorsiones y muchas veces no recordamos exactamente lo que experimentamos, y también que tenemos distintos almacenes donde guardamos cada información. Tanto el marketing como las políticas públicas tienen un efecto que depende del tipo de memoria al que apelen. Esto permite entender por qué muchas veces sube el dólar, por qué no funcionan las campañas de prevención del consumo de cigarrillos y alcohol y cómo lograr que las personas se comporten como los hacedores de políticas públicas desean.

Ante el inconveniente de la falta de información la pregunta es cómo se toman las decisiones en estos casos, ya que a raíz de información imperfecta los individuos presentan fallas en sus conductas, por ejemplo al momento de comprar una computadora probablemente juegue un papel muy importante el marketing y la emoción que cada marca genere en los consumidores, quienes tomarán la decisión no tanto por el costo del producto, sino por la satisfacción que le genere poseerlo.

La teoría clásica asume que el mercado se basa en la información perfecta. Según Simon esa perfección no existe. La información siempre es apropiada, valorada y sesgada de forma imperfecta.

También aquí es importante el conocimiento del proceso decisorio, ya que los consumidores podrían tener estrategias alternativas. Pero el sujeto no puede conocer directamente las consecuencias que se seguirán de su comportamiento. Si pudiese cono- cerlas operaría una especie de causalidad inversa: las consecuencias futuras serían las determinantes del comportamiento presente. Lo que hace es formar expectativas de las consecuencias futuras que están basadas en relaciones empíricas conocidas y en su información acerca de la situación existente.

El sujeto para actuar en este esquema con perfecta racionalidad tendría que disponer de una descripción completa de las consecuencias que se seguirían de cada estrategia alternativa y comparar estas consecuencias. Tendría que conocer en cada uno de sus aspectos qué cambios experimentaría el mundo si él se condujese de una manera o de otra, y tendría que seguir las consecuencias del comportamiento durante periodos ilimitados de tiempo. Por lo que sería inconcebible que el comportamiento real se acercara siquiera a la racionalidad (Simon, 1972).

Por otra parte se plantea el problema de los juicios de valor que aleja los análisis de los fenómenos económicos del ideal de objetividad, ya que somos miembros de sociedades con valores ideológicos que se aprenden y absorben. Estos juicios subjetivos muchas veces inconscientes influencian las cuestiones que los economistas estudian, los conceptos que emplean, las preguntas que hacen y las hipótesis que formulan (Mochón y Becker, 1997).

\section{Racionalidad limitada}

Todo lo desarrollado hasta el momento encuentra una buena síntesis en la Teoría de la Racionalidad Limitada de Simon. Ésta es en oposición a la teoría de la racionaliada "perfecta», que sabemos asume que, en una situación de decisión, la información y análisis personales son óptimos, y que las estimaciones de probabilidades son fácilmente realizables, sin presentar dificultades ni límites en los cálculos matemáticos que deben realizarse para determinar que la alternativa elegida es la mejor.

Pero Simon se esfuerza en demostrar que las decisiones reales de la gente son diferentes de las decisiones económicamente ideales, y que la economía 
es una teoría extremadamente formal que dice que las personas tenemos diversas preferencias que intentamos unir de forma coherente en la llamada función de utilidad. Por eso en su teoría de la racionalidad limitada ve el proceso de decisión desde un punto de vista diferente. En el proceso de toma de decisiones, e incluso en problemas relativamente simples, no se puede obtener un máximo ya que es imposible verificar todas las posibles alternativas. Las personas difieren tanto en oportunidades disponibles como en deseos, y muchas veces influenciados por factores de su entorno. Cuando un individuo debe decidir influyen en él tanto los deseos que posee como las oportunidades que él cree poseer. Es posible que el individuo no sea consciente de algunas oportunidades que en realidad le son viables, 0 puede creer que le son propicias ciertas oportunidades que en realidad no lo son, por lo tanto no puede garantizarse que elegirá la mejor alternativa.

Por estas cuestiones la racionalidad es limitada desde dos direcciones: desde el entorno del decisor, ya que no tiene acceso a la información perfecta, ni a la certidumbre e influyen en él factores exógenos como la cultura, las organizaciones en las que está inmerso etc., y desde el proceso mental del decisor pues este no tiene ni la estructura perfecta de preferencias, ni la capacidad completa de cálculo, y le afectan factores como la experiencia, la memoria, la percepción, las creencias y la sensibilidad personal (Universidad de Talca, s.f.).

La Teoría de la Racionalidad Limitada no asume al decisor como un ser no racional, sino un ser que trata de ser racional con lo que tiene. Se reconoce entonces la incapacidad de la teoría racional para captar completamente el proceso de decisión que llevan a cabo los individuos en la realidad.

En una entrevista el mismo Simon explica: «El término racionalidad limitada es, en mi concepción, ampliamente entendido como una advertencia a los economistas, de que no pueden predecir el comportamiento humano instalando un modelo abstracto de lo que es racional e infiriendo el comportamiento sobre eso. Hay que saber mucho sobre lo que está adentro de la cabeza de una persona y sobre los métodos que esa persona usa en sus cálculos. Y esas son cuestiones empíricas que no son para ser resueltas sentados en un sillón. Y mi crítica sobre la economía que sin dudas resumí con el término "racionalidad limitada" es que los economistas clásicos modernos piensan que ellos pueden construir teoría sin nunca preguntarse qué hacen realmente los seres humanos» (Simon, s.f.).

\section{Economía conductual}

Quien fue el Premio Nobel de Economía en 2002 Daniel Kahneman junto a su colaborador Amos Nathan Tversky realizaron investigaciones que dieron lugar a la denominada economía conductual. Se trata de una disciplina que combina la economía y la psicología, al investigar lo que ocurre en los mercados cuando los agentes sufren las limitaciones y complicaciones propias de los seres humanos.

Es una rama de la economía que en los últimos años ha revolucionado la manera de entender los procesos de toma de decisiones económicas, ya sea en lo particular como en el comportamiento humano en general. Ésta surge a partir de una aproximación inter y transdisciplinaria, diferenciándose del enfoque tradicional de la economía neoclásica. De acuerdo con la definición consignada en la Serie sobre Economía Conductual de la Universidad de Princeton, Estados Unidos, la economía conductual o también llamada economía del comportamiento «utiliza los hechos, modelos y métodos de ciencias relacionadas para determinar hallazgos descriptivamente exactos sobre la habilidad cognitiva humana y la interacción social, y para explorar las implicancias de estos hallazgos para la conducta económica. La más fértil de estas ciencias relacionadas ha sido la Psicología, pero la Sociología, la Antropología, la Biología y otros campos también pueden influir en la Economía de manera útil. La Economía Conductual se encuentra profundamente enraizada en hallazgos o métodos empíricos y hace que la economía avance a su manera — generando planteamientos teóricos, 
realizando predicciones más exactos sobre los fenómenos de campo, y sugiriendo mejores políticas» (Kosciuczyk, 2012:24).

Una parte importante de las contribuciones dentro de esta rama de la economía es la relación con la economía pública, por ejemplo al indagar cómo responden distintos tipos de sanciones o impuestos, 0 distintos fallos en el modo en el cual los individuos planifican el futuro que pueden tener implicaciones de interés en los diseños de las políticas públicas.

La teoría económica ha desarrollado desde hace varios años esfuerzos por explicar de la mejor manera las decisiones de los agentes económicos, con la finalidad de reconciliar los modelos teóricos con una gran cantidad de hallazgos empíricos que contradicen las predicciones de las teorías económicas convencionales. Entre estos esfuerzos destacan los planteamientos de la economía conductual con la finalidad de explicar conductas tan diversas como, por ejemplo, los hábitos poco saludables de alimentación, el exceso de confianza de los inversionistas bursátiles como también el comportamiento de compra del tipo «efecto manada» (Kosciuczyk, 2012).

Conjuntamente con los aportes interdisciplinarios, la economía conductual recurre sistemáticamente a la constatación experimental, en entornos controlados, de decisiones efectuadas por distintas muestras de la población (estudiantes de universidad, políticos, y empresarios). Esta metodología experimental fue desarrollada por los profesores Reinhard Selten (Premio Nobel de Economía 1996) y Vernon Smith.

\section{Economía experimental}

El premio Nobel de economía de 2002 fue otorgado conjuntamente a Vernon Smith y Daniel Kahneman. Los dos académicos, el primero economista y el segundo psicólogo, se han destacado por sus aportes a la economía empírica. Esto confirma por parte de la Real Academia Sueca que la economía ya no debe considerarse como una ciencia no experimental.
La Academia galardona a Smith por sus aportes fundamentales a la economía experimental y a Kahneman por sus contribuciones al estudio del comportamiento de los agentes aplicando el análisis de la psicología cognitiva.

La concesión del Nobel a Vernon Smith es un reconocimiento de la influencia de la economía experimental en el desarrollo de la economía como ciencia. Frente al método tradicional en el que las teorías se contrastan a partir de la observación del comportamiento de la economía real, la economía experimental permite analizar las proposiciones teóricas en el marco de un laboratorio, aproximando la economía a las ciencias naturales.

El primer experimento de Smith, An Experimental Study of Competitive Market Behavior, se considera como unas de las contribuciones más relevantes de la economía experimental. En él Smith informa sobre los resultados de una serie de juegos experimentales diseñados para contrastar las hipótesis de la teoría neoclásica de los mercados competitivos (Moreno García, 2002).

Este experimento aplica un método muy rudimentario utilizando a los alumnos de licenciatura; se divide a los alumnos en dos grupos: entre aquellos que representan la oferta y la demanda de un mercado; luego se distribuye a cada alumno un cartón con el precio de reserva, es decir, precio máximo (mínimo) de compra (o venta) que puede ofrecer para comprar (vender) el producto ficticio que se negocia. Los intercambios se realizan en distintos periodos de mercado consecutivos y mediante subasta en voz alta. El objetivo del alumno será conseguir el precio más favorable.

Desde sus experimentos preliminares Smith ya intenta reproducir las condiciones que se darían en un mercado real y no tanto las condiciones de competencia perfecta. De esta manera en el diseño mencionado no se cumplen los supuestos de múltiples participantes en el mercado ni los de información perfecta. El experimento supone un número reducido de participantes y se reproduce una situación en la que ni compradores ni vendedores 
conocen el precio de reserva del resto de participantes en el mercado, tan sólo pueden observar su comportamiento en el mercado. A través de subasta pública de precios en períodos consecutivos, lo que garantiza un proceso transparente, los participantes aprenden en el tiempo el comportamiento objetivo del resto de participantes.

Con el objetivo de reproducir el tipo de incentivos que se da en la economía real, los sujetos participantes en algunos de los ensayos reciben una remuneración que estará determinada por los beneficios ficticios que obtengan en su participación en el experimento.

Con el tiempo Smith incluye en sus experimentos nuevas condiciones de mercado y aspectos como comportamientos colusivos, control de precios y otras formas de intervención en los mercados. Luego los experimentos pudieron llevarse más lejos gracias a la ayuda de los ordenadores.

Uno de los principales aportes que la economía experimental hace a la ciencia económica es dotarla de un nuevo instrumento de análisis, siendo éste el experimento de laboratorio, y de un nuevo método que es el modelo experimental, para avanzar en el desarrollo de nuevas teorías.

El método experimental tiene además aplicaciones prácticas muy relevantes en el diseño de nuevos mercados. A través de la experimentación en el laboratorio el investigador puede analizar a priori qué tipo de institución es la más adecuada para el entorno del mercado en cuestión. El propio Smith fue contratado para analizar en el laboratorio el diseño de la Bolsa de Valores de Arizona, privatizaciones en Australia y Nueva Zelanda, y el mercado de agua de California (Moreno García, 2002).

Los experimentos que se han llevado a cabo permitieron extraer conclusiones teóricas que en muchos casos cuestionan los supuestos neoclásicos en los que se ha basado la ciencia económica. Entre ellos caben destacar tres proposiciones fundamentales: la relevancia de las instituciones, la irrelevancia de la información perfecta y de la racionalidad, y la inclusión del sentimiento de justicia en el comportamiento del agente.

\section{Críticas generales a la neuroeconomía}

Actualmente en aspectos económicos comienzan a utilizarse herramientas de las neurociencias. Es fascinante que se puedan analizar las conductas de los agentes económicos juntamente con la actividad cerebral, que es el gran motor de la humanidad.

Esto no quiere decir necesariamente que las teorías neoclásicas tengan que revisarse por completo, pero quizás cabe cuestionar su aplicación universal y la modificación de sus conclusiones a partir de la inclusión de agentes no racionales.

Quienes no promueven los avances en neuroeconomía se apoyan en que, si bien los modelos tradicionales económicos no describen a la perfección los comportamientos reales del mercado, no existen aún modelos neuroeconómicos desarrollados que permitan hacer un análisis de la economía y poder estudiar mediante ellos lo que ocurriría en el mercado en los próximos años. También atribuyen su postura a que esta nueva disciplina todavía no cuenta con fórmulas matemáticas que puedan explicar los comportamientos económicos.

Otra limitación es la imposibilidad de tener en cuenta todas las individualidades que aportan las neurociencias y la psicología. Ya que para formular modelos es necesario generalizar las conductas de los agentes, y considerar los aspectos amplios y mayoritarios de los comportamientos sociales.

\section{Reflexión final}

La economía no es una ciencia dura, y lejos de ello es tan particular que en un mismo año dos personas recibieron el Premio Nobel por decir lo contrario. Esto sucedió en 1974 cuando el galardón fue ganado por Gunar Myrdall, de la Escuela de Estocolmo, y por el ultraortodoxo Friedrieh Hayek (Campanario, 2005).

La neuroeconomía es resultado de una era moderna y globalizada, donde cada vez más las ciencias se entrelazan, se nutren entre ellas y podemos así llegar a nuevos descubrimientos. 
Mientras más sea la información que tengamos a disposición para entender el comportamiento estaremos en mejores condiciones de prever qué pasará en la economía.

Muchos economistas se muestran a favor de esta apertura, pero otros más escépticos opinan que el estudio de la activación de ciertas partes del cerebro no añade información en los análisis económicos. Otros están en una posición intermedia, no tan optimistas como los primeros, pero aceptan que la neuroeconomía aunque se enfrenta a ciertas limitaciones, ofrece aportes interesantes, sobre todo a la microeconomía. Estamos ante el comienzo de esta nueva disciplina, nuevas puertas se están abriendo y sin dudas aún queda mucho por descubrir.

\section{Referencias bibliográficas}

- Álvarez Gonzalez, M., y Trágapa Ortega, M. (2005). Principios de neurociencias para psicólogos. Buenos Aires: Paidós SAICF.

- Campanario, S. (2005). La economía de 10 insólito. Buenos Aires: Grupo Editorial Planeta SAIC.

- Kosciuczyk, V. (2012). El aporte de la Economía Conductual o Behavioural Economics a las Políticas Públicas: una aproximación al caso del consumidor real. Palermo Business Review, 18, 23-40.

- Manes, F. (2012). Lo que nos hace humanos: secretos del lóbulo frontal. TEDx Buenos Aires. Buenos Aires. Recuperado en noviembre 2016 de https://www.youtube.com/ watch? $=r 5 \mathrm{M} 018 \mathrm{pEkL} 4$.

(2015). Somos menos racionales de lo que creemos. Viva, 7. Recuperado en noviembre 2016 de http://www.clarin.com/viva/revista viva-facundo manes-neurocienciacerebro_0_HyPFWYwme.html

- Mochón, F., y Beker, V.A. (1997), Economía. Principios y aplicaciones. España: Mc Graw-Hill Interamericana de España SAU.
- Moreno García, P. (2002). Premio Nobel de Economía de 2002: Vernon Smith y Daniel Kahneman. Boletín Económico de ICE, 2749, 25-35. Recuperado de http://www.revistasice.com/CachePDF/BICE_2749_2535 6E2900A39EDBC0E1D892CB0 F0041B3A5.pdf

- Navarro, A.M. (2007). Neuroeconomía y metodología: algunas reflexiones iniciales. Buenos Aires: Academía Nacional de Ciencias de Buenos Aires.

- - (s.f.). Progresos recientes en neuroeconomía. Recuperado el 10/11/15 de http://a-navarro. com.ar/Papers/PROGRESOS\%20 RECIENTES\%20EN\%20NEUROECOMIA.pdf.

- Perticarari, N. R., y Hauque, S. M. (2013). Introducción a la Economía. Buenos Aires: La Ley S.A.E. e I.

- Simon, H.A. (1972). El comportamiento administrativo. Madrid: Aguilar SA.

- _- (1982). La nueva ciencia de la decisión gerencial. Buenos Aires: El Ateneo. (s.f.). Herbert Simon

(Entrevista - publicación año 2014). Recuperado en noviembre 2016 de You Tube: https://www.youtube.com/ watch? $v=z W-n 3 Q M-N L E$.

- Smith, A. (1941). Teoría de los Sentimientos Morales. España: El Colegio de México.

- Tetaz, M. (2014). Psychonomics. Buenos Aires: Ediciones B Argentina SA.

- Universidad de Talca (s.f.). Racionalidad perfecta y racionalidad limitada. Recuperado el 24/10/16 de - http://mggp.utalca.cl/docs/taller_ politicas_publicas/Racionalidad_Limitada.doc.

\section{Registro bibliográfico}

Arias, D.E. (2016). Análisis de Neuroeconomía como nuevo paradigma en la Ciencia Económica. Revista Ciencias Económicas, 13(02), 107-119. 